\title{
SCIAMACHY's View of the Polar Atmosphere
}

\author{
M. Gottwald, ${ }^{1}$ E. Krieg, ${ }^{1}$ C. von Savigny, ${ }^{2}$ S. Noël, ${ }^{2}$ P. Reichl, ${ }^{2}$ A. Richter, ${ }^{2}$ H. Bovensmann, ${ }^{2}$ and J. P. \\ Burrows $^{2}$
}

${ }^{1}$ Remote Sensing Technology Institute, German Aerospace Center, Oberpfaffenhofen, D-82234 Wessling, Germany (manfred.gottwald@dlr.de)
${ }^{2}$ Institute of Environmental Physics, University of Bremen, Otto-Hahn-Allee 1, D-28359 Bremen, Germany (csavigny@iup.physik.uni-bremen.de)

Abstract The instrument SCIAMACHY onboard the European ENVISAT mission provides unique capabilities for deriving atmospheric geophysical parameters. Since its launch in early 2002 it has operated successfully in orbit. Due to ENVISAT's high inclination orbit the polar regions are monitored continuously. We report here results about the status of the polar atmosphere in the past 5 years with special emphasis on the southern hemisphere. This part of the atmosphere is considered to be highly sensitive to anthropogenic impacts on the Earth system and thus to climate change. The acquired data permit retrieving information on the Earth's atmosphere from troposphere up to the mesosphere.

Citation: Gottwald, M., E. Krieg, C. von Savigny, S. Noël, P. Reichl, A. Richter, H. Bovensmann, and J. P. Burrows (2007), SCIAMACHY's View of the Polar Atmosphere, in Antarctica: A Keystone in a Changing World - Online Proceedings of the $10^{\text {th }}$ ISAES, edited by A. K. Cooper and C. R. Raymond et al., USGS Open-File Report 2007-1047, Short Research Paper 011, 4 p.; doi:10.3133/of2007-1047.srp011.

\section{SCIAMACHY on ENVISAT}

The SCIAMACHY instrument (SCanning Imaging Absorption SpectroMeter for Atmospheric CHartographY) is part of the payload of ESA's ENVISAT mission (Gottwald et al., 2006). SCIAMACHY has been provided jointly by the Deutsches Zentrum für Luft- und Raumfahrt (DLR) and the Nederlands Instituut voor Vliegtuigontwikkeling en Ruimtevaart (NIVR) with contributions from the Belgisch Instituut voor RuimteAëronomie (BIRA). The main scientific objective of the SCIAMACHY mission is to improve our knowledge of global atmospheric change by observing a large number of geophysical parameters including e.g. the concentration of trace gases such as $\mathrm{O}_{3}, \mathrm{BrO}, \mathrm{HCHO}, \mathrm{NO}_{2}, \mathrm{SO}_{2}, \mathrm{OClO}$, $\mathrm{H}_{2} \mathrm{O}, \mathrm{CO}, \mathrm{CO}_{2}, \mathrm{CH}_{4}$ and properties of aerosols and clouds.

The hosting ENVISAT platform (Fig. 1) is ESA's largest Earth Observation satellite built and operated so far. It travels in a sun-synchronous orbit with a mean altitude of $800 \mathrm{~km}$, an inclination of $98.5^{\circ}$ and an orbital period of $100.6 \mathrm{~min}$. The descending node crossing time corresponds to $10 \mathrm{a} . \mathrm{m}$. local time.

\section{The Instrument}

SCIAMACHY is an absorption spectrometer for the ultraviolet (UV) to the short-wave infrared (SWIR) range between 214 and $2386 \mathrm{~nm}$ (Bovensmann et al., 1999). Sunlight scattered and reflected off the Earth's atmosphere is dispersed into 8 detector channels with a spectral resolution sufficiently high to identify and analyze absorption features caused by atmospheric trace gases. Radiation from the atmosphere enters the spectrograph via the elevation scan mirror (nadir) or azimuth plus elevation scan mirror (limb, solar and lunar occultation). For calibration and monitoring purposes, solar or lunar light can also be collected from above the atmosphere. After being directed onto the entrance slit, the light is successively split up into several wavelength channels. Finally the dispersed light reaches the detectors. Detectors $1-5(=214-1063 \mathrm{~nm})$ cover the UV to near infrared (NIR) range and detectors 6-8 $(=971-2386 \mathrm{~nm})$ the SWIR range. Several components as e.g. a Polarisation Measurement Device, a spectral line source and a white light source can be used for calibrating the recorded spectra.

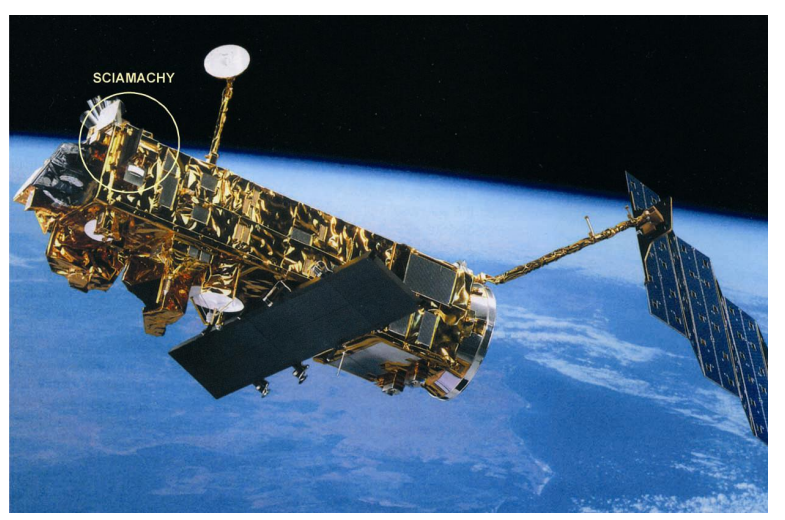

Figure 1. Artist's impression of SCIAMACHY on ENVISAT.

\section{The Operation Concept}

SCIAMACHY is accommodated on ENVISAT's front panel looking into flight direction. This determines, together with the selected sun-synchronous orbit, the observation conditions of the sun and the moon in occultation and the geolocation of limb measurements.

To achieve the scientific goals, the instrument observes the scattered and reflected spectral radiance in nadir and limb (Fig. 2) geometry, the spectral radiance transmitted through the atmosphere in solar and lunar occultation geometry and the extraterrestrial solar irradiance and the lunar radiance. This results in a variety of line-of-sight geometries permitting to derive not only total column densities of trace gases from nadir measurements but also profile information from limb and occultation observations. In nadir mode the atmospheric volume at the subsatellite point is scanned to yield total column trace gas densities (from top to bottom of the atmosphere). In limb mode the subsequent horizontal 
scans step up from the horizon to the top of the atmosphere thus providing vertically resolved profiles. SCIAMACHY operates continuously along the orbit. Each orbit between in-orbit sunrise and sunset the polar regions are always within the coverage of nadir and limb scans for $>20 \mathrm{~min}$.

For retrieving trace gas information from absorption spectra it is of prime importance to record spectra with good signal-to-noise ratios. The spectrograph's exposure parameters can be adjusted to the expected light levels along the orbit in order to account for the usually high solar zenith distances, i.e. low light levels, when crossing the polar regions. Current retrieval methods have become rather robust such that even spectra obtained under those conditions provide detailed insight into the chemistry and physics of the polar atmosphere.

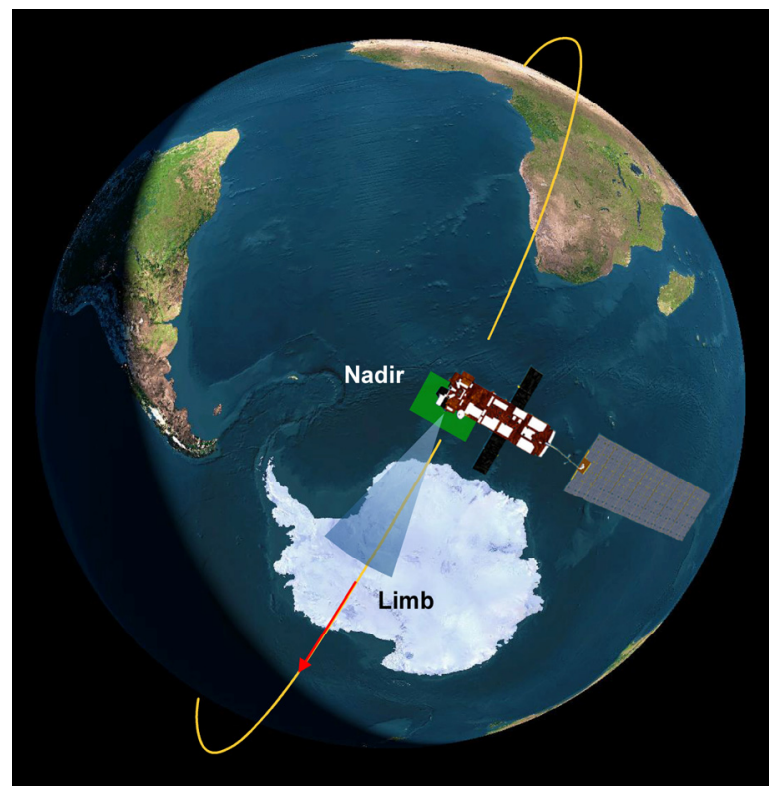

Figure 2. SCIAMACHY's nadir (green) and limb (blue) viewing geometries.

\section{The Earth's Atmosphere at the Poles}

The Earth's atmosphere above the polar caps exhibits several phenomena not observed at low latitude regions. Caused by their remote location, polar regions do not show high concentrations of tropospheric polluting trace gases or particles. However this 'cleanliness' makes them vulnerable to external perturbations such that the polar atmosphere is considered a good indicator for global atmospheric change. The stratosphere over Antarctica had created high public interest with the detection of the ozone hole end of the past century. Its explanation had not only illustrated anthropogenic impact onto our environment but also emphasized the extreme conditions found at high altitudes over the South Pole. In winter, when the polar vortex is strongest, the atmosphere becomes isolated and the trapped air cools down to less than $-80{ }^{\circ} \mathrm{C}$ which is a pre-requisite for the development of Polar Stratospheric Clouds (PSC) and associated ozone depletion. This phenomenon makes Antarctica unique since over the north polar region the vortex is much less pronounced, i.e. temperatures in the north polar stratosphere less frequently reach the low levels required for the creation of PSCs. Obtaining a detailed view of the polar atmosphere is crucial for the understanding of the global role of the atmosphere in the Earth system. In the following sections we present examples of SCIAMACHY measurements over the polar regions demonstrating the unique capabilities of spaceborne remote sensing over such remote areas.

\section{The Troposphere}

One unique feature of the polar troposphere is the regular occurrence of episodes with almost completely depleted ozone in the surface layers. This phenomenon which is observed in both hemispheres in polar spring is related to the so called bromine explosion in which reactive bromine levels are increasing exponentially over a short period of time, efficiently destroying all ozone. The source of this natural phenomenon is bromine from sea salt, probably released in heterogeneous processes on the surface of first year ice and/or frost flowers forming on fresh ice. The most abundant bromine substance during these events is $\mathrm{BrO}$ which can be measured with SCIAMACHY using the retrieval developed for GOME (Richter et al., 1998) after adaptation to SCIAMACHY (Afe et al., 2004).

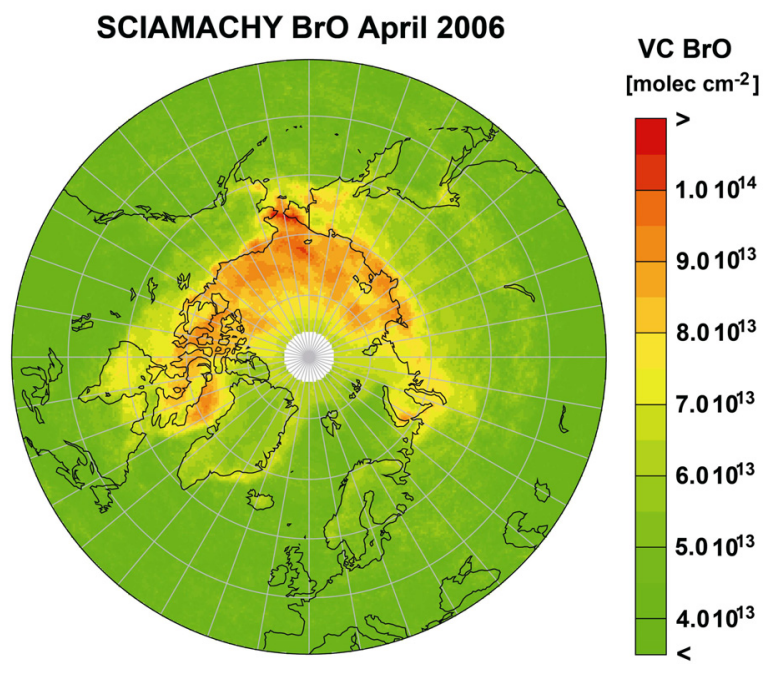

Figure 3. Polar map of $\mathrm{BrO}$ total column for April 2006 as observed by SCIAMACHY. Values above $5 \times 10^{13}$ $\mathrm{molec} / \mathrm{cm}^{2}$ are indicative of enhanced tropospheric $\mathrm{BrO}$.

This is illustrated in Fig. 3, where $\mathrm{BrO}$ columns are shown in the northern hemisphere in April 2006. As can be seen, enhanced $\mathrm{BrO}$ is found over most of the polar ice cap with the highest values along the shores of the Arctic Sea. This is related to the release mechanism of $\mathrm{BrO}$ which needs both ice surfaces and access to open water for sea salt aerosol. 


\section{The Stratosphere}

SCIAMACHY's observations allow the retrieval of several stratospheric key species such as $\mathrm{O}_{3}, \mathrm{NO}_{2}, \mathrm{BrO}$, and within the polar vortex under twilight conditions also OClO. Several of these species are of great interest in terms of stratospheric $\mathrm{O}_{3}$ chemistry, since they participate in the catalytic $\mathrm{O}_{3}$ destruction cycles. In nadir observation mode total vertical column can be retrieved, whereas the limb observation mode permits the retrieval of vertical profiles.

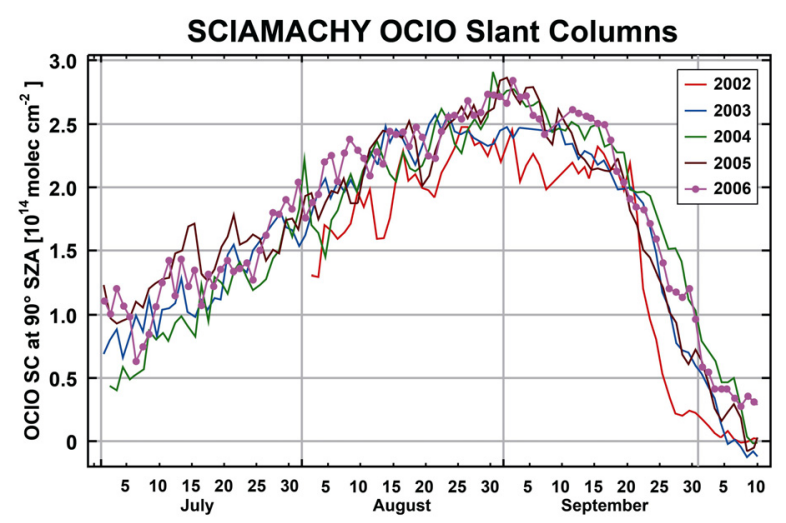

Figure 4. OClO slant columns, i.e. column along the mean light path through the atmosphere, at $90^{\circ} \mathrm{SZA}$ as retrieved from measurements of the SCIAMACHY instrument over Antarctica from 2002-2006. Data analysis is similar to that applied for GOME (Richter et al., 2005).

An example for the ability of SCIAMACHY to monitor the polar stratosphere in nadir observation mode is shown in Fig. 4, where OClO columns are displayed for different years. OClO is a good indicator of chlorine activation since it is mainly formed by reaction of $\mathrm{ClO}$ with $\mathrm{BrO}$. $\mathrm{As} \mathrm{OClO}$ is rapidly photolysed in sunlight, data from observations at $90^{\circ}$ solar zenith angle (SZA) are presented. After an initial increase from July to early September, OClO columns rapidly decrease until early October when they fall below the detection limit of the instrument. The variability in the values is linked to dynamical displacements of the polar vortex; the only year with significantly different activation levels in the Antarctic vortex was 2002, the year of the split vortex. This is in contrast to the situation in the northern Hemisphere, where interannual variation in chlorine activation is largely depending on stratospheric temperatures.

Apart from minor constituents SCIAMACHY is also capable to detect and map polar stratospheric clouds. PSCs are of crucial importance for the formation of the Antarctic ozone hole, because heterogeneous reactions in and on the PSC particles lead to the transformation of $\mathrm{Cl}$ reservoir species to chemically more active species. PSCs are detected in SCIAMACHY measurements of limbscattered sunlight using a colour-ratio approach.

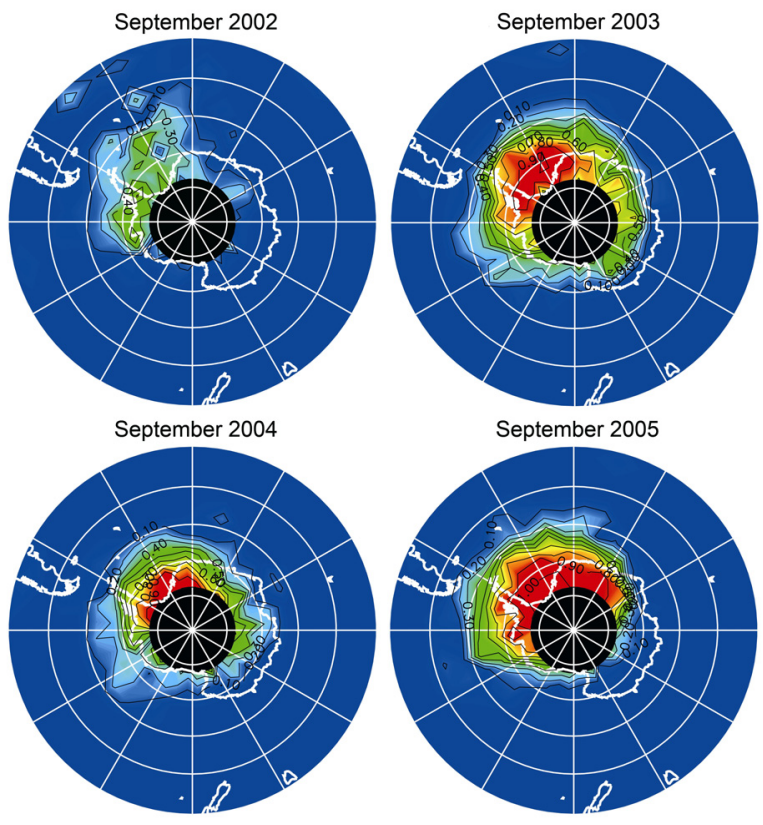

Figure 5. Monthly mean maps of the polar stratospheric cloud (PSC) occurrence rates in the southern hemisphere for the years 2002 to 2005 .

Fig. 5 shows monthly mean Antarctic PSC occurrence rate maps for the month of September of the years 2002 to 2005. September typically has the largest PSC occurrence rates in a given year. Fig. 5 illustrates that there is also significant variability from year to year. Particularly in 2002 the PSC occurrence rates are low. This is related to the unexpectedly early major stratospheric warming associated with the split of the polar vortex after September 22, 2002. After the end of September, there were basically no PSCs observed anymore over the southern polar cap region.

\section{The Mesosphere}

SCIAMACHY also allows the global retrieval of several key species in the mesosphere (e.g., $\mathrm{O}_{3}, \mathrm{NO}, \mathrm{Mg}$, $\mathrm{Fe}$ ) as well as noctilucent clouds (NLC) which are also known as polar mesospheric clouds (PMC). Here we present NLCs in more detail. NLCs consist of $\mathrm{H}_{2} \mathrm{O}$ ice particles with radii smaller than $100 \mathrm{~nm}$ and occur poleward of about $50^{\circ}$ latitude in the summer hemisphere at altitudes of $82-85 \mathrm{~km}$. They are discussed as early indicators of global change, because their formation and existence depends sensitively on the thermal conditions and the abundance of $\mathrm{H}_{2} \mathrm{O}$ in the polar mesopause region. Long-term satellite measurements of the NLC nadir reflectivity with SBUV instruments indeed show a long term increase, apart from a pronounced solar cycle variation. NLCs are easily detected in SCIAMACHY limb measurements, because they scatter solar radiation efficiently. Furthermore, NLC particle sizes can be derived from SCIAMACHY limb spectra in the UV spectral range. For wavelengths below about $300 \mathrm{~nm}$ 
multiple scattering is negligible and the limb spectra are simply determined by the solar irradiance spectrum and the spectral dependence of the NLC scattering crosssection (von Savigny et al., 2007a). Fig. 6 shows as an example the NLC occurrence rates for different latitude bands during southern hemisphere NLC seasons and the latitudinal variation of derived NLC radii for northern hemisphere measurements in July 2005.
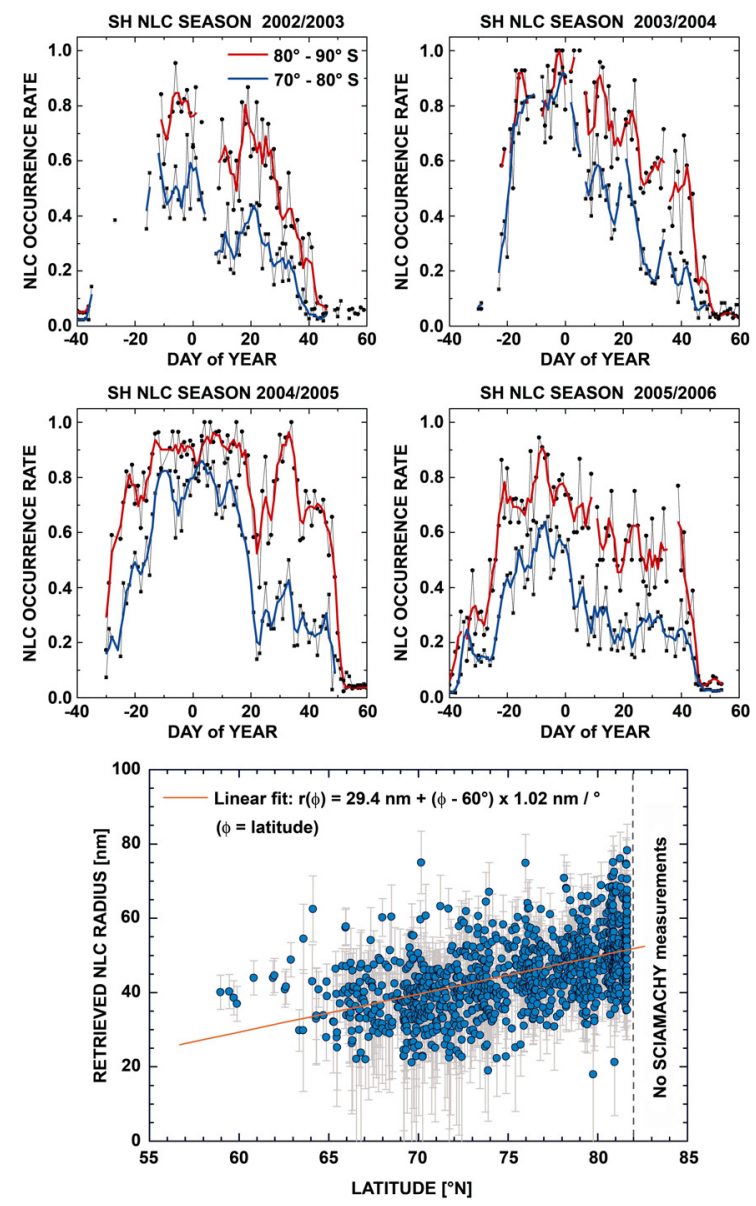

Figure 6. Top panel: Zonally averaged NLC occurrence rates between $70^{\circ} \mathrm{S}$ and $80^{\circ} \mathrm{S}$ (blue) and $80^{\circ} \mathrm{S}$ and $90^{\circ} \mathrm{S}$ (red) for different years. The thick solid lines are 3-day running mean values. Bottom panel: Latitudinal variation of NLC radii for July 2005 derived from SCIAMACHY measurements assuming a log-normal NLC particle size distribution with $\sigma=1.4$.

Noteworthy is the rapid decrease in the NLC occurrence rate during the 2004/2005 NLC season around day 15 , which coincides exactly with strongly enhanced precipitation of solar energetic protons caused by a solar proton event (von Savigny et al., 2007b). For the $\left[70^{\circ} \mathrm{S}\right.$, $\left.80^{\circ} \mathrm{S}\right]$-latitude band the NLC occurrence rate drops from about $80 \%$ on January 15 to about $15 \%$ on January 21 . The disappearance is caused by a simultaneous increase in mesopause temperatures, but the exact mechanisms leading to the temperature increase and the subsequent disappearance of NLCs are not fully established. The NLC radii - shown in the bottom panel of Fig. $6-$ increase with latitude which is a consequence of the lower mesopause temperatures at higher latitudes. The derived radii are in good agreement with most of the previously retrieved NLC particle radii (von Savigny et al., 2007b).

\section{Summary}

SCIAMACHY observed the polar atmosphere in the past 5 years in great detail. We present several examples of geophysical parameters retrieved from these measurements. Starting in the troposphere we discuss interactions between the arctic ocean and tropospheric trace gases. One layer up in the stratosphere catalytic ozone depletion is still a major concern with several participating key species being monitored continuously. During the ozone hole episodes PSCs are detected and yield insight into the underlying chemical processes. Finally the mesosphere hosts NLCs which are considered to be an early indicator of global change. SCIAMACHY's measurements allow not only identification of NLCs but also the retrieval of their particle sizes. The polar atmosphere is considered to be highly sensitive to anthropogenic impacts on the Earth system and thus to climate change. SCIAMACHY's measurements contribute significantly to the understanding of the underlying atmospheric chemistry and transport processes.

Acknowlegements. This work was supported by the German Ministry of Education and Research (BMBF) and the German Aerospace Center (DLR) under grant 50EE0027, as well as by the University of Bremen. SCIAMACHY is jointly funded by Germany, the Netherlands, and Belgium. The authors also wish to thank the editorial team and the reviewers; their efforts have helped to make this research paper possible.

\section{References}

Afe, O. T., A. Richter, B. Sierk, F. Wittrock and J. P. Burrows (2004), $\mathrm{BrO}$ Emission from Volcanoes - a Survey using GOME and SCIAMACHY Measurements, Geophys. Res.Lett., 31, L24113, doi:10.1029/2004GL020994.

Bovensmann, H., J. P. Burrows, M. Buchwitz, J. Frerick, S. Noël, V. V. Rozanov, K. V. Chance, and A. P. H. Goede (1999), SCIAMACHY: Mission objectives and measurement modes, J. Atmos. Sci., 56(2), 127-150.

Gottwald, M., H. Bovensmann, G. Lichtenberg et al. (2006), SCIAMACHY, Monitoring the Changing Earth's Atmosphere, DLRIMF.

Richter, A., F. Wittrock, M. Weber, S. Beirle, S. Kühl, U. Platt, T. Wagner, W. Wilms-Grabe, and J. P. Burrows (2005), GOME observations of stratospheric trace gas distributions during the splitting vortex event in the Antarctic winter 2002 Part I: Measurements, J. Atmos. Sci., 62 (3), 778-785.

Richter, A., F. Wittrock, M. Eisinger and J. P. Burrows (1998), GOME observations of tropospheric $\mathrm{BrO}$ in Northern Hemispheric spring and summer 1997, Geophys. Res. Lett., No. 25, 2683-2686.

von Savigny, C., and J. P. Burrows (2007a), Latitudinal variation of NLC particle radii derived from northern hemisphere SCIAMACHY/Envisat limb measurements, Adv. Space Res., in press, doi:10.1016/ j.asr.2006.12.032.

von Savigny, C., M. Sinnhuber, H. Bovensmann, J. P. Burrows, M.-B. Kallenrode, and M. J. Schwartz (2007b), On the Disappearance of Noctilucent Clouds during the January 2005 Solar Proton Events, Geophys. Res. Lett., 34, L02805, doi:10.1029/2006GL02810 